MATHEMATICS OF COMPUTATION

Volume 74, Number 250, Pages 723-742

S $0025-5718(04) 01675-8$

Article electronically published on May 18, 2004

\title{
SOME PROPERTIES OF THE GAMMA AND PSI FUNCTIONS, WITH APPLICATIONS
}

\author{
S.-L. QIU AND M. VUORINEN
}

\begin{abstract}
In this paper, some monotoneity and concavity properties of the gamma, beta and psi functions are obtained, from which several asymptotically sharp inequalities follow. Applying these properties, the authors improve some well-known results for the volume $\Omega_{n}$ of the unit ball $B^{n} \subset \mathbb{R}^{n}$, the surface area $\omega_{n-1}$ of the unit sphere $S^{n-1}$, and some related constants.
\end{abstract}

\section{INTRODUCTION}

For real and positive values $x$ and $y$, the Euler gamma function, the beta and psi (or polygamma) functions are defined as

$$
\Gamma(x)=\int_{0}^{\infty} t^{x-1} e^{-t} d t, \quad B(x, y)=\frac{\Gamma(x) \Gamma(y)}{\Gamma(x+y)}, \quad \psi(x)=\frac{\Gamma^{\prime}(x)}{\Gamma(x)},
$$

respectively. For the extensions to complex variables and for the basic properties of these functions, see $\mathrm{AS}$, AAR, [Mi], [T], and [WW. Over the past half century, many authors have obtained various properties and inequalities for these very important functions (see [A1], $\mathrm{A} 2], \mathrm{A} 5], \mathrm{G}], \mathrm{Ke}, \mathrm{K2}],[\mathrm{L}], \mathrm{MSC}$ and bibliographies therein).

Formulas for the volumes of geometric bodies sometimes involve the gamma function. This topic and related inequalities have been studied recently in AQ, [A1]- $\mathrm{A} 4], \mathrm{BP}$, [EL]. Let $B^{n}$ and $S^{n-1}$ be the unit ball and unit sphere in $\mathbb{R}^{n}$, respectively, $\Omega_{n}$ be the volume of $B^{n}$, and $\omega_{n-1}$ denote the surface area of $S^{n-1}$. Set $\Omega_{0}=1$. It is well known that $\Omega_{n}$ is increasing for $2 \leq n \leq 5$ and decreasing for $n \geq 5$, while $\omega_{n-1}$ is increasing for $2 \leq n \leq 7$ and decreasing for $n \geq 7$ (see [BH] pp. 263-264] and [AVV1, p. 38]):

$$
\Omega_{n}=\frac{2 \pi}{n} \Omega_{n-2}=\frac{\pi^{n / 2}}{\Gamma(1+n / 2)}, \quad \omega_{n-1}=n \Omega_{n}=2 \frac{\pi^{n / 2}}{\Gamma(n / 2)} .
$$

Throughout this paper, we let $\gamma=0.57721 \cdots=-\psi(1)$ denote the EulerMascheroni constant, $(2 n-1) ! !=(2 n-1)(2 n-3) \cdots 1$ for $n \in \mathbb{N}=\{k ; k$ is a natural number\},

$$
I_{n}=\int_{0}^{\pi / 2} \sin ^{n-2} t d t, \quad J_{n}=\int_{0}^{\pi / 2} \sin ^{(2-n) /(n-1)} t d t,
$$

Received by the editor April 2, 2002 and, in revised form, September 27, 2003.

2000 Mathematics Subject Classification. Primary 33B15; Secondary 26B15, 26D15, 51M25.

Key words and phrases. Gamma function, beta function, psi function, monotoneity, concavity, inequalities. 


$$
\begin{gathered}
A_{n}=\left(\frac{\omega_{n-1}}{2^{n} c_{n}}\right)^{1 /(n-1)}, B_{n}=b_{n}^{n-1}, D_{n}=\frac{1}{n-1}\left(\frac{\omega_{n-1}}{c_{n}}\right)^{1 /(n-1)}, \\
b_{n}=J_{n} /(n-1) \text { and } c_{n}=\left(2 J_{n}\right)^{1-n} \omega_{n-2},
\end{gathered}
$$

for $n \in \mathbb{N}$ with $n \geq 2$. These constants have applications in some fields of mathematics such as geometry of Grassmannian subspaces of $\mathbb{R}^{n}[\mathrm{KR}$, optimization theory [B], geometric function theory [AVV1 pp. 234-246], [V], [Vu], as well as geometry of spaces of constant curvature $[\mathrm{BH}]$.

These are some examples of the fields where the gamma function is frequently used. The accumulated literature on the gamma function is so vast that it is difficult for someone working chiefly in these specialized areas of research to find the property of the gamma function which, in this particular application, would lead to the desired conclusion. This is exactly how we were led to explore the gamma function, our main research area being geometric function theory; see [V], [Vu] [AVV1], AQ]. Some of the latest developments on special functions that have been studied in this context are reviewed in AVV2. The statement of some of our main results, which we think have independent interest as such, now follows.

Theorem A. For $n=1,2,3, \ldots$

$$
2 \sqrt{\frac{\pi}{2 n+\pi-2}} \leq \frac{\Omega_{n}}{\Omega_{n-1}}<2 \sqrt{\frac{\pi}{2 n+1}} .
$$

Theorem B. For $x \geq 1$, let $P(x)=\max \{\sqrt{\pi x}, \sqrt{4 x-1}\}$. Then for $x \geq 1$

$$
x^{(1-\gamma) x-1} P(x) \leq \Gamma(x) P(x) \leq 2 \Gamma(x+1 / 2) .
$$

Theorem C. For $x>1$

$$
\log x-\frac{1}{2 x}-\frac{1}{12 x^{2}}<\psi(x)<\log x-\frac{1}{2 x}-\frac{2 \gamma-1}{2 x^{2}} .
$$

Theorems A, B, and C are special cases of the more technical Theorems 1.17, 1.12 , and 2.1, respectively. We next proceed to review some earlier results and to discuss our preliminary and main results.

Various properties of $\Omega_{n}$ and $\omega_{n-1}$ have been proved in these contexts. For example, it was shown in $[\mathrm{B}]$ that for $n \geq 3$,

$$
\sqrt{\frac{2 \pi}{n+1}} \leq \frac{\Omega_{n}}{\Omega_{n-1}} \leq \sqrt{\frac{2 \pi}{n}}
$$

and

$$
\sqrt{\frac{2 \pi}{n-1}} \leq \frac{\omega_{n-1}}{\omega_{n-2}} \leq \sqrt{\frac{2 \pi}{n-2}}
$$

and a new property of $\Omega_{n}$ was obtained in $\mathrm{AQ}$. Several inequalities for $A_{n}, B_{n}, c_{n}$, $D_{n}, I_{n}$ and $J_{n}$ were shown or stated in AVV1. For example, it was proved that for $n \geq 3$,

$$
\begin{gathered}
\sqrt{\frac{\pi}{2 n-2}}<I_{n}<\sqrt{\frac{\pi}{2 n-4}}, \\
-1<(n-1)\left(J_{n}-n+1-\log 2\right)<1-(\pi / 2) \log 2<0, \\
\lim _{n \rightarrow \infty} A_{n} / n=1, \lim _{n \rightarrow \infty} B_{n}=\lim _{n \rightarrow \infty} D_{n}=2,
\end{gathered}
$$


and

$$
1<B_{n}<2 \text { for } n \geq 2 \text {. }
$$

(See AVV1, pp. 42-43].)

It is the purpose of the present paper to show some properties of $\Gamma(x), B(x, y)$ and $\psi(x)$ and then, based on these properties, to improve some well-known results for $\Omega_{n}, \omega_{n-1}, A_{n}, B_{n}, b_{n}, I_{n}$ and $J_{n}$, such as (1.6)-(1.11). We now state some of the main results of this paper below.

1.12. Theorem. (1) The function $f_{1}(x) \equiv \Gamma(x+1 / 2) /[\sqrt{x} \Gamma(x)]$ is strictly increasing and log-concave from $(0, \infty)$ onto $(0,1), f_{2}(x) \equiv f_{1}(1 / x)$ is log-convex on $(0, \infty)$, and $f_{3}(x) \equiv 1 / f_{1}(x)$ is convex on $(0, \infty)$.

(2) The function $f_{4}(x) \equiv x-[\Gamma(x+1 / 2) / \Gamma(x)]^{2}=x\left[1-f_{1}(x)^{2}\right]$ is strictly increasing and concave from $(0, \infty)$ onto $(0,1 / 4)$. Moreover, for $x \geq 1$,

$$
\begin{aligned}
& x^{(1-\gamma) x-1} P(x) \leq \Gamma(x) P(x) \leq 2 \Gamma(x+1 / 2) \\
& \leq \sqrt{\pi x} \Gamma(x)(2 / \sqrt{\pi})^{1-1 / x} \leq \sqrt{\pi x}(2 / \sqrt{\pi})^{1-1 / x} x^{x-1 / 2},
\end{aligned}
$$

with equality in each instance iff $x=1$, where $P(x)=\max \{\sqrt{\pi x}, \sqrt{4 x-1}\}$. The third and the fourth inequalities in (1.13) are asymptotically sharp as $x$ tends to $\infty$.

(3) For each fixed $x>0$, define functions $f_{5}, f_{6}$ and $f_{7}$ on $(0,1)$ by

$$
f_{5}(s)=\frac{1}{1-s}\left[\log x-\frac{1}{s} \log \frac{\Gamma(x+s)}{\Gamma(x)}\right], f_{6}(s)=\frac{\beta(x)-f_{5}(s)}{s}, f_{7}(s)=\frac{s\left[f_{5}(s)-\alpha(x)\right]}{1-s},
$$

respectively, where $\alpha(x)=\psi(x+1)-\log x$ and $\beta(x)=\log x-\psi(x)$. Then $f_{5}$ and $f_{6}$ are both strictly decreasing on $(0,1)$ with ranges $(\alpha(x), \beta(x))$ and $(\xi(x), \eta(x))$, respectively, while $f_{7}$ is strictly increasing from $(0,1)$ onto $(0, \theta(x))$, where

$$
\xi(x)=\beta(x)-\alpha(x), \eta(x)=\frac{1}{2} \psi^{\prime}(x)-\beta(x) \quad \text { and } \quad \theta(x)=\alpha(x)-\frac{1}{2} \psi^{\prime}(x+1) .
$$

In particular, for $x>0$ and $s \in(0,1)$,

$$
\begin{gathered}
\exp \{s(s-1) \beta(x)\}<\exp \{s(1-s) \rho(s, x)\}<\frac{\Gamma(x+s)}{x^{s} \Gamma(x)} \\
<\exp \{s(s-1) \omega(s, x)\} \leq \exp \{s(s-1) \alpha(x)\},
\end{gathered}
$$

where

and

$$
\rho(s, x)=\max \{s \xi(x)-\beta(x),-\alpha(x)+(1-s) \theta(x) / s\}
$$

$$
\omega(s, x)=\min \{s \eta(x)-\beta(x),-\alpha(x)\} .
$$

The above theorem yields some inequalities of $\Gamma(x)$. Some similar or related results have been proved recently in $\mathrm{AQ}$, [BP], [EL], [A5], [K2], [Me1]-[Me3].

1.15. Remarks. (1) For $n \in \mathbb{N}$, let $G(n, k)$ be the so-called Grassmannian, and let $\tau_{n}$ denote the invariant measure on $G(n, 1)$, that is, on the set of all straight lines through the origin (cf. [KR p. 206]). Set $g(n)=\tau_{n}(G(n, 1)$ ). Then the functions $f_{1}(x), f_{4}(x)$ and $f_{5}(s)$ in Theorem 1.12 are related to $g(n)$. Therefore, Theorem 1.12 is related to some results in KR. For example, it was proved in KR, p. 214] that $g(n)$ is increasing for $n \in \mathbb{N}$, while Theorem $1.12(1)$ says that $g(n) / \sqrt{n}$ is also strictly increasing for $n \in \mathbb{N}$ since $g(n)=\sqrt{n \pi / 2} f_{1}(n / 2)$ by the expression of $g(n)$ (see [KR p. 214]). 
(2) The double inequality (1.14) and its Corollary 3.2 are related to a result in [LL], which gives the estimates

$$
(1-s) \log (x+s / 2)<D_{s}(x)<(1-s) \log \left(x+(\Gamma(s))^{1 /(s-1)}\right)
$$

for $s \in(0,1)$ and $x>0$, where $D_{s}(x)=\log \Gamma(x+1)-\log \Gamma(x+s)$. For some other related results, see [A3, p. 365], [Me1], MSC], etc.

1.16. Theorem. (1) The function $g_{1}(x) \equiv[x B(x, 1 / 2)]^{1 / x}$ is strictly decreasing and convex from $(0, \infty)$ onto $(1,4)$.

(2) There exists a unique $x_{1} \in(1.5,2)$ such that the function

$$
g_{2}(x) \equiv \frac{1}{2 x+1}\left[\frac{1}{2} B\left(x, \frac{1}{2}\right)\right]^{1 /(2 x)} B\left(\frac{1}{4 x}, \frac{1}{2}\right),
$$

for $x \in(0, \infty)$, is strictly decreasing on $\left(0, x_{1}\right]$ and increasing on $\left[x_{1}, \infty\right)$, with $g_{2}(1 / 2)=\pi^{2} / 4$ and $\lim _{x \rightarrow \infty} g_{2}(x)=2$.

(3) There exists a unique $x_{2} \in(33,34)$ such that the function

$$
g_{3}(x) \equiv 2^{1 /(2 x)}\left(1+\frac{1}{2 x}\right) g_{2}(x)
$$

for $x \in(0, \infty)$, is strictly decreasing on $\left(0, x_{2}\right]$ and increasing on $\left[x_{2}, \infty\right)$, with $g_{3}(1 / 2)=\pi^{2}$ and $\lim _{x \rightarrow \infty} g_{3}(x)=2$.

As an example of the applications of the above two theorems, we shall prove the following improvements upon some results for $\Omega_{n}, \omega_{n-1}, A_{n}, B_{n}, b_{n}, I_{n}$ and $J_{n}$.

1.17. Theorem. (1) For $n \in \mathbb{N} \backslash\{1,2\}$, the function $F_{1}(n) \equiv 2 n-\pi I_{n}^{-2}$ is strictly increasing with $F_{1}(3)=6-\pi$ and $\lim _{n \rightarrow \infty} F_{1}(n)=3$. In particular, for $n \geq 3$,

$$
\sqrt{\frac{\pi}{2 n-(6-\pi)}} \leq I_{n}<\sqrt{\frac{\pi}{2 n-3}}
$$

with equality iff $n=3$.

(2) The function $F_{2}(n) \equiv n-2 \pi\left[\omega_{n-2} / \omega_{n-1}\right]^{2}$ is strictly increasing for $n \in$ $\mathbb{N} \backslash\{1,2\}$, with $F_{2}(3)=3-(\pi / 2)$ and $\lim _{n \rightarrow \infty} F_{2}(n)=3 / 2$. In particular, for $n \geq 3$,

$$
2 \sqrt{\frac{\pi}{2 n-6+\pi}} \leq \frac{\omega_{n-1}}{\omega_{n-2}}<2 \sqrt{\frac{\pi}{2 n-3}},
$$

with equality iff $n=3$.

(3) The function $F_{3}(n) \equiv n-2 \pi\left[\Omega_{n-1} / \Omega_{n}\right]^{2}$ is strictly increasing for $n \in \mathbb{N}$, with $F_{3}(1)=1-\pi / 2$ and $\lim _{n \rightarrow \infty} F_{3}(n)=-1 / 2$. In particular, for $n \in \mathbb{N}$,

$$
2 \sqrt{\frac{\pi}{2 n+\pi-2}} \leq \frac{\Omega_{n}}{\Omega_{n-1}}<2 \sqrt{\frac{\pi}{2 n+1}},
$$

with equality iff $n=1$.

(4) Let $n \in \mathbb{N}$. Then $b_{n}$ is strictly decreasing for $n \geq 2$, and $B_{n}$ is strictly increasing for $n \geq 2$. In particular, for $n \geq 2$,

$$
\frac{\pi}{2} \leq B_{n}<2, \quad(n-1)\left(\frac{\pi}{2}\right)^{1 /(n-1)} \leq J_{n} \leq(n-1) \min \left\{2^{1 /(n-1)}, \frac{\pi}{2}\right\}
$$

with equality in each instance iff $n=2$. 
(5) Let $a=1-\log 2, b=3-0.5 B(1 / 4,1 / 2)=0.37794 \cdots, c=\left[\pi^{2}-3(\log 4)^{2}\right] / 24$ $=0.171007 \cdots$, and $n \in \mathbb{N}$. Then the function $F_{4}(n) \equiv(n-1)\left(n-J_{n}-a\right)$ is strictly increasing for $n \geq 3$, with $F_{4}(3)=d \equiv 2(b-a)=0.14217 \cdots$ and $\lim _{n \rightarrow \infty} F_{4}(n)=c$. In particular, for $n \geq 3$,

$$
d \leq(n-1)\left(n-J_{n}-a\right)<c,
$$

with equality iff $n=3$.

(6) Let $n \in \mathbb{N}$. Then the function $F_{5}(n) \equiv A_{n} / n$ is strictly decreasing for $2 \leq n \leq 5$ and increasing for $n \geq 5$. In particular,

$$
\lim _{n \rightarrow \infty} \frac{1}{n} A_{n}=1, C \leq \frac{1}{n} A_{n} \begin{cases}\leq A_{2} / 2=\pi^{2} / 8, & \text { for } 2 \leq n \leq 5, \\ <1, & \text { for } n \geq 5\end{cases}
$$

where

$$
C=\frac{1}{5} A_{5}=\frac{\sqrt{\pi}}{10}\left(\frac{2}{3}\right)^{1 / 4} \frac{\Gamma(1 / 8)}{\Gamma(5 / 8)}=0.8411396629 \cdots .
$$

(7) Let $n \in \mathbb{N}$. Then $D_{n}$ is strictly decreasing for $2 \leq n \leq 69$ and increasing for $n \geq 69$. In particular,

$$
\lim _{n \rightarrow \infty} D_{n}=2, D \leq D_{n} \begin{cases}\leq D_{2}=\pi^{2}, & \text { for } 2 \leq n \leq 69 \\ <2, & \text { for } n \geq 69\end{cases}
$$

where

$$
D=D_{69}=\frac{\sqrt{2 \pi}}{68}\left(\frac{33 !}{67 ! !}\right)^{1 / 68} \frac{\Gamma(1 / 136)}{\Gamma(69 / 136)}=1.9853487779 \cdots
$$

\section{Some properties of $\psi(x)$}

In this section, we obtain some properties of $\psi(x)$, which are also needed in the proofs of the theorems stated in Section 1.

2.1. Theorem. (1) The function $h_{1}(x) \equiv \psi(x+1 / 2)-\psi(x)-[1 /(2 x)]$ is strictly decreasing and convex from $(0, \infty)$ onto $(0, \infty)$. Moreover, $h_{2}(x) \equiv h_{1}(1 / x)$ is convex on $(0, \infty)$.

(2) The function $h_{3}(x) \equiv x h_{1}(x)$ is strictly decreasing from $(0, \infty)$ onto $(0,1 / 2)$.

(3) The function $h_{4}(x) \equiv x^{2} h_{1}(x)$ is strictly increasing from $(0, \infty)$ onto $(0,1 / 8)$. In particular, for $x>1$,

$$
\psi(x)+\frac{1}{2 x}+\frac{1.5-\log 4}{x^{2}}<\psi\left(x+\frac{1}{2}\right)<\psi(x)+\frac{1}{2 x}+\frac{1}{8 x^{2}} .
$$

(4) The function $h_{5}(x) \equiv x^{2}[\psi(x)-\log x]+(x / 2)$ is strictly decreasing and convex from $(0, \infty)$ onto $(-1 / 12,0)$. In particular, for $x>1$,

$$
\log x-\frac{1}{2 x}-\frac{1}{12 x^{2}}<\psi(x)<\log x-\frac{1}{2 x}-\frac{2 \gamma-1}{2 x^{2}} .
$$

(5) Let $\eta$ and $\theta$ be functions defined in Theorem 1.12(3). Then $\eta$ and $\theta$ are both strictly decreasing from $(0, \infty)$ onto $(0, \infty)$. 
Proof. (1) Differentiation gives

$$
h_{1}^{\prime}(x)=-\frac{h_{6}(x)}{x^{2}} \equiv \psi^{\prime}\left(x+\frac{1}{2}\right)-\psi^{\prime}(x)+\frac{1}{2 x^{2}}=\frac{1}{2 x^{2}}-\int_{0}^{\infty} \frac{t e^{-x t}}{1+e^{-t / 2}} d t,
$$

where

since [AS, 6.4.1]

$$
h_{6}(x)=\int_{0}^{\infty} \frac{u e^{-u} d u}{1+e^{-u /(2 x)}}-\frac{1}{2},
$$

$$
\psi^{(n)}(x)=(-1)^{n+1} \int_{0}^{\infty} \frac{t^{n} e^{-x t}}{1-e^{-t}} d t .
$$

Clearly, $h_{6}$ is strictly decreasing from $(0, \infty)$ onto $(0,1 / 2)$. Hence $h_{1}^{\prime}$ is strictly increasing from $(0, \infty)$ onto $(-\infty, 0)$, and the monotoneity and convexity of $h_{1}$ follow.

By [AS, 6.3.5], $h_{1}(x)=\psi(x+1 / 2)-\psi(x+1)+[1 /(2 x)]$ so that $h_{1}\left(0^{+}\right)=\infty$. The limiting value $\lim _{x \rightarrow \infty} h_{1}(x)=0$ follows from [AS, 6.3.18].

The convexity of $h_{2}$ follows from the monotoneity of $h_{6}$, since

$$
h_{2}^{\prime}(x)=-h_{1}^{\prime}(1 / x) / x^{2}=h_{6}(1 / x) .
$$

(2) Put $y=1 / x$. Then

$$
h_{3}(x)=\frac{h_{2}(y)}{y} \quad \text { and } \quad \frac{h_{2}^{\prime}(y)}{\frac{d}{d y}(y)}=h_{2}^{\prime}(y),
$$

and hence, the monotoneity of $h_{3}$ follows from the convexity of $h_{2}$ and the Monotone l'Hôpital's Rule [AVV1, Theorem 1.25].

By [AS, 6.3.5], $h_{3}(x)$ can be written as

$$
h_{3}(x)=\frac{x}{2}\left[2 \psi\left(x+\frac{1}{2}\right)-\psi(x+1)-\psi(x)\right],
$$

and hence, $h_{3}\left(0^{+}\right)=1 / 2$. It follows from [AS, 6.3.18] that $\lim _{x \rightarrow \infty} h_{3}(x)=0$.

(3) Write $h_{4}(x)=h_{2}(y) / y^{2}$, where $y=1 / x$, and let

$$
h_{7}(y)=h_{6}\left(\frac{1}{y}\right)=\int_{0}^{\infty} \frac{u e^{-u} d u}{1+e^{-u y / 2}}-\frac{1}{2}, \quad h_{8}(y)=y .
$$

Then $h_{7}(0)=h_{8}(0)=0$, and by $(2.4)$,

$$
\begin{gathered}
\frac{h_{2}^{\prime}(y)}{\frac{d}{d y}\left(y^{2}\right)}=\frac{h_{7}(y)}{2 h_{8}(y)}, \\
\frac{h_{7}^{\prime}(y)}{h_{8}^{\prime}(y)}=h_{9}(y) \equiv \frac{1}{2} \int_{0}^{\infty} \frac{u^{2} e^{-u(1+y / 2)}}{\left(1+e^{-u y / 2}\right)^{2}} d u .
\end{gathered}
$$

Clearly, $h_{9}$ is strictly decreasing in $y$ on $(0, \infty)$. Hence the monotoneity of $h_{4}$ follows from the Monotone l'Hôpital's Rule AVV1 Theorem 1.25].

Clearly, $h_{4}\left(0^{+}\right)=\lim _{x \rightarrow 0} x h_{3}(x)=0$. By l'Hôpital's Rule, (2.5) and (2.6),

$$
\lim _{x \rightarrow \infty} h_{4}(x)=\lim _{y \rightarrow 0} \frac{h_{2}(y)}{y^{2}}=h_{9}(0)=\frac{1}{16} \int_{0}^{\infty} u^{2} e^{-u} d u=\frac{1}{8} .
$$

Inequality $(2.2)$ is clear. 
(4) Using [AS, 6.3.21], we obtain by differentiation

$$
\begin{aligned}
h_{5}^{\prime}(x)= & h_{10}(x) \equiv 2 x[\psi(x)-\log x]+x^{2}\left[\psi^{\prime}(x)-\frac{1}{x}\right]+\frac{1}{2} \\
= & -2 x\left[\frac{1}{2 x}+\int_{0}^{\infty} \frac{2 t d t}{\left(t^{2}+x^{2}\right)\left(e^{2 \pi t}-1\right)}\right]+\frac{1}{2} \\
& +x^{2}\left[\frac{1}{2 x^{2}}+\int_{0}^{\infty} \frac{4 x t d t}{\left(t^{2}+x^{2}\right)^{2}\left(e^{2 \pi t}-1\right)}\right] \\
= & 4 x^{3} \int_{0}^{\infty} \frac{t d t}{\left(t^{2}+x^{2}\right)^{2}\left(e^{2 \pi t}-1\right)}-4 x \int_{0}^{\infty} \frac{t d t}{\left(t^{2}+x^{2}\right)\left(e^{2 \pi t}-1\right)} \\
= & -4 x \int_{0}^{\infty} \frac{t^{3} d t}{\left(t^{2}+x^{2}\right)^{2}\left(e^{2 \pi t}-1\right)}=-4 x \int_{0}^{\infty} \frac{u^{3} d u}{\left(1+u^{2}\right)^{2}\left(e^{2 \pi u x}-1\right)},
\end{aligned}
$$

where $u=t / x$. Since $x \longmapsto x /\left(e^{2 \pi u x}-1\right)$ is strictly decreasing on $(0, \infty), h_{10}$ is strictly increasing on $(0, \infty)$. Hence the monotoneity and convexity of $h_{5}$ follow.

Clearly, $h_{5}\left(0^{+}\right)=0, h_{5}(1)=(1 / 2)-\gamma$. By [AS, 6.3.18], $h_{5}(\infty)=-1 / 12$.

(5) By differentiation and (2.4), we get

$$
\begin{aligned}
2 x \eta^{\prime}(x) & =2 x\left[\frac{1}{2} \psi^{\prime \prime}(x)-\frac{1}{x}+\psi^{\prime}(x)\right]=\int_{0}^{\infty} \frac{x t(2-t) \mathrm{e}^{-x t}}{1-\mathrm{e}^{-t}} d t-2 \\
& =\frac{1}{x} \int_{0}^{\infty} \frac{u(2-u / x) \mathrm{e}^{-u}}{1-\mathrm{e}^{-u / x}} d u-2,
\end{aligned}
$$

which is strictly increasing from $(0, \infty)$ onto $(-\infty, 0)$ since the function $v \mapsto$ $v(2-v) /\left(1-\mathrm{e}^{-v}\right)$ is strictly decreasing from $(0, \infty)$ onto $(-\infty, 2)$. This yields the monotoneity of $\eta$.

By [AS, 6.3.5], we can rewrite $\eta(x)$ as

$$
\eta(x)=\frac{1}{2} \psi^{\prime}(x+1)+\frac{1}{2 x^{2}}-\log x+\psi(x+1)-\frac{1}{x},
$$

from which we see that $\eta\left(0^{+}\right)=\infty$. The limiting value $\lim _{x \rightarrow \infty} \eta(x)=0$ follows from [AS, 6.3.18 and 6.4.12].

Next, similarly, we have

$$
2 x \theta^{\prime}(x)=\int_{0}^{\infty} \frac{x t(2+t) \mathrm{e}^{-(x+1) t}}{1-\mathrm{e}^{-t}} d t-2=\frac{1}{x} \int_{0}^{\infty} \frac{u(2+u / x) \mathrm{e}^{-u}}{\mathrm{e}^{u / x}-1} d u-2,
$$

which is strictly increasing from $(0, \infty)$ onto $(-2,0)$ since $v \mapsto v(2+v) /\left(\mathrm{e}^{v}-1\right)$ is strictly decreasing from $(0, \infty)$ onto $(0,2)$. Hence the monotoneity of $\theta$ follows.

Clearly, $\theta\left(0^{+}\right)=\infty$. By [AS, 6.3.18 and 6.4.12], we obtain $\lim _{x \rightarrow \infty} \theta(x)=0$.

2.7. Remarks. (1) The double inequality (2.3) improves the inequality obtained in [ABRVV, Theorem 3.1].

(2) It is well known that [AS, 6.1.3]

$$
\gamma=\lim _{n \rightarrow \infty} d_{n}, \quad d_{n}=\sum_{k=1}^{n} \frac{1}{k}-\log n,
$$

and by $[\mathrm{AS}, 6.3 .2]$,

$$
d_{n}-\gamma=\psi(n+1)-\log n
$$


S. R. Tims and J. A. Tyrrell [TT] obtained the bounds

$$
\frac{1}{2(n+1)}<d_{n}-\gamma<\frac{1}{2(n-1)}, \quad n \geq 2,
$$

for $d_{n}-\gamma$, which was improved by R. M. Young [Y] as

$$
\frac{1}{2(n+1)}<d_{n}-\gamma<\frac{1}{2 n}, \quad \text { for } \quad n \in \mathbb{N}
$$

while G. D. Anderson et al. ABRVV proved

$$
\frac{1-\gamma}{n}<d_{n}-\gamma<\frac{1}{2 n}, \quad \text { for } \quad n \in \mathbb{N}
$$

Recently, H. Alzer [A3, Theorem 3] established the double inequality

$$
\frac{1}{2(n+\alpha)} \leq d_{n}-\gamma<\frac{1}{2(n+\beta)}, \quad \text { for } \quad n \in \mathbb{N},
$$

where $\alpha=\{1 /[2(1-\gamma)]\}-1=0.1826 \cdots$ and $\beta=1 / 6$. Let $h_{5}$ be as in Theorem 2.1(4). Then

$$
h_{5}(n)=n^{2}\left\{\psi(n+1)-\log n-\frac{1}{n}\right\}+\frac{n}{2}=n^{2}\left(d_{n}-\gamma\right)-\frac{n}{2},
$$

and hence our Theorem 2.1(4) implies the following estimates for $d_{n}-\gamma$ :

2.13. Corollary. For $n \in \mathbb{N}$,

$$
\frac{1}{2 n}-\frac{\alpha}{n^{2}}<d_{n}-\gamma \leq \frac{1}{2 n}-\frac{\beta}{n^{2}}
$$

with equality iff $n=1$, where the constants $\alpha=1 / 12=0.08333 \cdots$ and $\beta=$ $\gamma-1 / 2=0.07721 \cdots$ are best possible.

Some further results on the approximation of $\gamma$ are given in [K1].

\section{Proofs of the Main theorems}

In this section we prove the theorems stated in Section 1.

3.1. Proof of Theorem 1.12. (1) Logarithmic differentiation gives

$$
f_{1}^{\prime}(x) / f_{1}(x)=h_{1}(x)
$$

where $h_{1}$ is as in Theorem 2.1(1), and hence the monotoneity and log-concavity of $f_{1}$ follow from Theorem 2.1(1). By [AS, 6.1.15 and 6.1.37], we have

$$
f_{1}\left(0^{+}\right)=\lim _{x \rightarrow 0} \sqrt{x} \frac{\Gamma(x+1 / 2)}{\Gamma(x+1)}=0
$$

and

$$
\lim _{x \rightarrow \infty} f_{1}(x)=\lim _{x \rightarrow \infty} \frac{e^{-(x+1 / 2)}(x+1 / 2)^{x}}{\sqrt{x} e^{-x} x^{x-1 / 2}}=1 .
$$

Since $f_{2}^{\prime}(x) / f_{2}(x)=-h_{4}(1 / x)$, where $h_{4}$ is as in Theorem 2.1(3), the logconvexity of $f_{2}$ follows from Theorem 2.1(3).

Next, by differentiation,

$$
f_{3}^{\prime}(x)=-f_{1}^{\prime}(x) / f_{1}(x)^{2}=-h_{1}(x) / f_{1}(x),
$$

which is strictly increasing on $(0, \infty)$ by Theorem $2.1(1)$, and hence the convexity of $f_{3}$ follows. 
(2) Set $y=\Gamma(x+1 / 2) / \Gamma(x)$. Then

$$
f_{4}^{\prime}(x)=1-2 y^{2}\left[\psi\left(x+\frac{1}{2}\right)-\psi(x)\right]
$$

and

where

$$
\frac{1}{2 y^{2}} f_{4}^{\prime \prime}(x)=-\left[\psi\left(x+\frac{1}{2}\right)-\psi(x)\right]^{2} f_{8}(x)
$$

$$
\begin{gathered}
f_{8}(x)=f_{9}^{\prime}(x)=2+\frac{\psi^{\prime}(x+1 / 2)-\psi^{\prime}(x)}{[\psi(x+1 / 2)-\psi(x)]^{2}}, \\
f_{9}(x)=2 x-\left[\psi\left(x+\frac{1}{2}\right)-\psi(x)\right]^{-1}=2 \frac{h_{4}(x)}{h_{3}(x)+1 / 2}
\end{gathered}
$$

and $h_{3}$ and $h_{4}$ are as in Theorem 2.1. By Theorem 2.1(2)-(3), $f_{9}$ is strictly increasing from $(0, \infty)$ onto $(0,1 / 2)$, so that $f_{8}(x)>0$ for $x \in(0, \infty)$, and hence $f_{4}^{\prime \prime}(x)<0$ for $x \in(0, \infty)$. This yields the concavity of $f_{4}$. Furthermore, by part (1) and Theorem $2.1(2)$,

$$
\begin{aligned}
f_{4}^{\prime}(x) & >\lim _{x \rightarrow \infty} f_{4}^{\prime}(x) \\
& =1-2 \lim _{x \rightarrow \infty} f_{1}(x)^{2} \cdot x\left[\psi\left(x+\frac{1}{2}\right)-\psi(x)\right] \\
& =1-2\left[\lim _{x \rightarrow \infty} f_{1}(x)\right]^{2}\left[\lim _{x \rightarrow \infty} h_{3}(x)+\frac{1}{2}\right]=0
\end{aligned}
$$

for $x \in(0, \infty)$, and hence the monotoneity of $f_{4}$ follows.

By part (1), $f_{4}\left(0^{+}\right)=\lim _{x \rightarrow 0}\left[x-x f_{1}(x)^{2}\right]=0$. Applying l'Hôpital's Rule, we obtain

$$
\begin{aligned}
\lim _{x \rightarrow \infty} f_{4}(x) & =\lim _{x \rightarrow \infty} \frac{1-f_{1}(x)^{2}}{1 / x}=\lim _{t \rightarrow 0} \frac{1-f_{2}(t)^{2}}{t} \\
& =-2 \lim _{t \rightarrow 0} f_{2}(t) f_{2}^{\prime}(t)=2 \lim _{t \rightarrow 0} f_{2}(t)^{2} h_{4}(1 / t) \\
& =2 \lim _{x \rightarrow \infty} f_{1}(x)^{2} h_{4}(x)=1 / 4 .
\end{aligned}
$$

The second and third inequalities in (1.13) follow from the monotoneity of $f_{4}$ on $(0, \infty)$ and the log-convexity of $f_{2}$ on $(0,1)$, while the first and fourth inequalities in (1.13) hold by [AQ Theorem 1.5]. The equality case is clear. The asymptotic sharpness of the third and fourth inequalities in (1.13) follows from the monotoneity of $f_{1}$ and $\mathrm{AQ}$, Theorem 1.5].

(3) For $x>0$, let

$$
\begin{gathered}
f_{10}(s)=\log x-\frac{1}{s} \log \frac{\Gamma(x+s)}{\Gamma(x)}, \quad f_{11}(s)=1-s, \\
f_{12}(s)=s \psi(x+s)-\log \Gamma(x+s)+\log \Gamma(x) \quad \text { and } \quad f_{13}(s)=s^{2} .
\end{gathered}
$$

Then $f_{5}(s)=f_{10}(s) / f_{11}(s), f_{10}(1)=f_{11}(1)=f_{12}(0)=f_{13}(0)=0$, and

$$
\frac{f_{10}^{\prime}(s)}{f_{11}^{\prime}(s)}=\frac{f_{12}(s)}{f_{13}(s)}, \quad \frac{f_{12}^{\prime}(s)}{f_{13}^{\prime}(s)}=\frac{1}{2} \psi^{\prime}(x+s) .
$$

Since $\psi^{\prime}(t)$ is strictly decreasing on $(0, \infty)$, the monotoneity of $f_{5}$ follows from the Monotone l'Hôpital's Rule [AVV1, Theorem 1.25]. 
By l'Hôpital's Rule,

$$
f_{5}\left(1^{-}\right)=\lim _{s \rightarrow 1} \frac{f_{12}(s)}{f_{13}(s)}=\psi(x+1)-\log x=\alpha(x),
$$

and

$$
\begin{aligned}
f_{5}\left(0^{+}\right) & =f_{10}\left(0^{+}\right)=\log x-\lim _{s \rightarrow 0} \frac{\log \Gamma(x+s)-\log \Gamma(x)}{s} \\
& =\log x-\psi(x)=\beta(x) .
\end{aligned}
$$

Let $f_{14}(s)=s \beta(x)-s f_{5}(s), f_{15}(s)=s^{2}$,

$$
\begin{aligned}
f_{16}(s)= & \psi^{\prime}(x+s)(1-s)^{2}-2\{[\log x-\psi(x+s)](1-s) \\
& +s \log x-\log \Gamma(x+s)+\log \Gamma(x)\},
\end{aligned}
$$

and $f_{17}(s)=(1-s)^{3}$. Then $f_{6}(s)=f_{14}(s) / f_{15}(s), f_{14}(0)=f_{15}(0)=f_{14}^{\prime}(0)=$ $f_{15}^{\prime}(0)=f_{16}(1)=f_{17}(1)=0$ and

$$
\frac{f_{14}^{\prime \prime}(s)}{f_{15}^{\prime \prime}(s)}=\frac{f_{16}(s)}{2 f_{17}(s)}, \quad \frac{f_{16}^{\prime}(s)}{f_{17}^{\prime}(s)}=-\frac{1}{3} \psi^{\prime \prime}(x+s) .
$$

It is well known that $\psi^{\prime \prime}$ is strictly increasing on $(0, \infty)$. Hence the monotoneity of $f_{6}$ follows from the Monotone l'Hôpital's Rule [AVV1, Theorem 1.25].

By l'Hôpital's Rule, we get

$$
f_{6}\left(0^{+}\right)=\lim _{s \rightarrow 0} \frac{f_{14}^{\prime}(s)}{f_{15}^{\prime}(s)}=\lim _{s \rightarrow 0} \frac{f_{14}^{\prime \prime}(s)}{f_{15}^{\prime \prime}(s)}=\frac{f_{16}(0)}{2 f_{17}(0)}=\frac{1}{2} \psi^{\prime}(x)-\beta(x)=\eta(x) .
$$

The limiting value $f_{6}\left(1^{-}\right)$follows from the result for $f_{5}$.

Next, let

$$
f_{18}(s)=s \log x-\log \frac{\Gamma(x+s)}{\Gamma(x)}-s(1-s) \alpha(x) \quad \text { and } \quad f_{19}(s)=(1-s)^{2} .
$$

Then $f_{7}(s)=f_{18}(s) / f_{19}(s), f_{18}(1)=f_{19}(1)=f_{18}^{\prime}(1)=f_{19}^{\prime}(1)=0$ and

$$
\frac{f_{18}^{\prime \prime}(s)}{f_{19}^{\prime \prime}(s)}=\alpha(x)-\frac{1}{2} \psi^{\prime}(x+s),
$$

which is strictly increasing in $s$ on $(0,1)$ by the well-known monotoneity of $\psi^{\prime}$. Hence the monotoneity of $f_{7}$ follows from the Monotone l'Hôpital's Rule AVV1. Theorem 1.25].

Clearly, $f_{7}\left(0^{+}\right)=0$. By l'Hôpital's Rule, $f_{7}\left(1^{-}\right)=\theta(x)$.

The inqualities in (1.14) are clear.

3.2. Corollary. (1) For $x>1$ and $s \in(0,1)$,

$$
x^{s} \exp \left\{\frac{s(s-1)}{2 x}\left(1+\frac{1}{6 x}\right)\right\}<\frac{\Gamma(x+s)}{\Gamma(x)}<x^{s} \exp \left\{\frac{s(s-1)}{2 x}\left(1-\frac{1}{6 x}\right)\right\} .
$$

(2) For $s \in[0,1)$, let $\lambda(s)=1+1 /[3(1-s)]$ and let $\beta$ be as in Theorem 1.12(3). Then for $x>(1-s) / 2$ and $s \in(0,1)$,

$$
\frac{\Gamma(x+s)}{\Gamma(x)}>x^{s} \exp \left\{-s(1-s) \beta\left(\frac{1-s}{2}\right)\right\}>x^{s} \exp \{-s \lambda(s)\} .
$$


Proof. By (2.3) and [AS, 6.3.5], we have

$$
\beta(x)<\frac{1}{2 x}\left(1+\frac{1}{6 x}\right) \text { and } \alpha(x)>\frac{1}{2 x}\left(1-\frac{1}{6 x}\right),
$$

and hence part (1) follows from (1.14)

Next, by (2.4),

$$
x \beta^{\prime}(x)=1-x \psi^{\prime}(x)=1-\int_{0}^{\infty} \frac{x t \mathrm{e}^{-x t}}{1-\mathrm{e}^{-t}} d t=1-\frac{1}{x} \int_{0}^{\infty} \frac{u \mathrm{e}^{-u} d u}{1-\mathrm{e}^{-u / x}},
$$

which is strictly increasing from $(0, \infty)$ onto $(-\infty, 0)$ since $v \mapsto v /\left(1-\mathrm{e}^{-v}\right)$ is strictly increasing from $(0, \infty)$ onto $(1, \infty)$. Hence $\beta$ is strictly decreasing and convex from $(0, \infty)$ onto itself. This, together with the first inequality in (2.3), yields (3.3).

\subsection{Proof of Theorem 1.16. (1) By (1.1), we can rewrite $g_{1}(x)$ as}

$$
g_{1}(x)=\left[\frac{\sqrt{\pi} x \Gamma(x)}{\Gamma(x+1 / 2)}\right]^{1 / x}=\left[\frac{\sqrt{\pi} \Gamma(x+1)}{\Gamma(x+1 / 2)}\right]^{1 / x} .
$$

Logarithmic differentiation gives

$$
g_{1}^{\prime}(x) / g_{1}(x)=g_{4}(x) \equiv g_{5}(x) / g_{6}(x),
$$

where $g_{6}(x)=x^{2}$ and

$$
g_{5}(x)=x\left[\psi(x+1)-\psi\left(x+\frac{1}{2}\right)\right]+\log \frac{\Gamma(x+1 / 2)}{\sqrt{\pi} \Gamma(x+1)},
$$

with $g_{5}(0)=g_{6}(0)=0, \lim _{x \rightarrow \infty} g_{5}(x)=-\infty$, and

$$
\frac{g_{5}^{\prime}(x)}{g_{6}^{\prime}(x)}=g_{7}(x) \equiv \frac{1}{2}\left[\psi^{\prime}(x+1)-\psi^{\prime}\left(x+\frac{1}{2}\right)\right] .
$$

Since $\psi^{\prime \prime}(x)$ is strictly increasing, $g_{7}^{\prime}(x)>0$. Hence $g_{4}$ is strictly increasing on $(0, \infty)$ by the Monotone l'Hôpital's Rule [AVV1, Theorem 1.25]. By l'Hôpital's Rule, (3.5), (3.6), and AS, 6.4.2, 6.4.4, 6.4.10 and 23.2.24],

$$
g_{4}\left(0^{+}\right)=g_{7}\left(0^{+}\right)=\frac{1}{2}\left[\psi^{\prime}(1)-\psi^{\prime}\left(\frac{1}{2}\right)\right]=-\zeta(2)=-\frac{\pi^{2}}{6}
$$

and

$$
\lim _{x \rightarrow \infty} g_{4}(x)=\lim _{x \rightarrow \infty} g_{7}(x)=0 .
$$

Here $\zeta(x)$ is the Riemann zeta function. Hence $g_{4}$ is strictly increasing from $(0, \infty)$ onto $\left(-\pi^{2} / 6,0\right)$, so that the monotoneity of $g_{1}$ follows from (3.5). Since $g_{1}^{\prime}(x)=$ $-g_{1}(x) \cdot\left[-g_{4}(x)\right], g_{1}^{\prime}$ is strictly increasing on $(0, \infty)$ and the convexity of $g_{1}$ follows.

By l'Hôpital's Rule and [AS, 6.3.2, 6.3.3 and 6.3.18],

$$
\begin{aligned}
g_{1}\left(0^{+}\right) & =\exp \left\{\lim _{x \rightarrow 0} \frac{\log \Gamma(x+1)-\log \Gamma(x+1 / 2)+(\log \pi) / 2}{x}\right\} \\
& =\exp (\psi(1)-\psi(1 / 2))=4
\end{aligned}
$$

and

$$
\lim _{x \rightarrow \infty} g_{1}(x)=\exp \left\{\lim _{x \rightarrow \infty}\left[\psi(x+1)-\psi\left(x+\frac{1}{2}\right)\right]\right\}=1 .
$$


(2) By (1.1) and [AS, 6.1.15], $g_{2}(x)$ can be rewritten as

$$
g_{2}(x)=\frac{\sqrt{\pi} \Gamma(1+1 /(4 x))}{\Gamma((3 / 2)+1 /(4 x))}\left[\frac{\sqrt{\pi}}{2} \frac{\Gamma(x)}{\Gamma(x+1 / 2)}\right]^{1 /(2 x)} .
$$

Logarithmic differentiation gives

$$
\begin{aligned}
& \frac{4 x^{2} g_{2}^{\prime}(x)}{g_{2}(x)}=g_{8}(x) \equiv 2 x\left[\psi(x)-\psi\left(x+\frac{1}{2}\right)\right] \\
& \quad-2 \log \frac{\sqrt{\pi} \Gamma(x)}{2 \Gamma(x+1 / 2)}+\psi\left(\frac{1}{4 x}+\frac{3}{2}\right)-\psi\left(\frac{1}{4 x}+1\right) .
\end{aligned}
$$

Since

$$
g_{8}^{\prime}(x)=2 x\left[\psi^{\prime}(x)-\psi^{\prime}\left(x+\frac{1}{2}\right)\right]+\frac{1}{4 x^{2}}\left[\psi^{\prime}\left(\frac{1}{4 x}+1\right)-\psi^{\prime}\left(\frac{1}{4 x}+\frac{3}{2}\right)\right],
$$

which is positive for $x \in(0, \infty)$ by the monotoneity of $\psi^{\prime}$ on $(0, \infty), g_{8}$ is strictly increasing on $(0, \infty)$.

Let $h_{3}$ be as in Theorem 2.1. Then $g_{8}$ can be rewritten as

$$
g_{8}(x)=-2 h_{3}(x)-1-2 \log \frac{\sqrt{\pi} \Gamma(x)}{2 \Gamma(x+1 / 2)}+\psi\left(\frac{1}{4 x}+\frac{3}{2}\right)-\psi\left(\frac{1}{4 x}+1\right) .
$$

Therefore, by [AS, 6.3.18 and 6.1.37],

$$
\begin{aligned}
g_{8}\left(0^{+}\right)= & -2+\log 4+\lim _{x \rightarrow 0}\left\{2 \log x+\log \left[\left(\frac{1}{4 x}+\frac{3}{2}\right) /\left(\frac{1}{4 x}+1\right)\right]\right. \\
& \left.-\frac{1}{[1 /(2 x)]+3}+\frac{1}{[1 /(2 x)]+2}\right\}=-\infty
\end{aligned}
$$

and

$$
\begin{aligned}
\lim _{x \rightarrow \infty} g_{8}(x) & =-1+\psi\left(\frac{3}{2}\right)-\psi(1)-2 \log \frac{\sqrt{\pi}}{2}-2 \lim _{x \rightarrow \infty} \log \frac{\Gamma(x)}{\Gamma(x+1 / 2)} \\
& =1-\log \pi-2 \lim _{x \rightarrow \infty} \log \left[\sqrt{\frac{e}{x}}\left(1+\frac{1}{2 x}\right)^{-x}\right]=\infty .
\end{aligned}
$$

Hence $g_{8}$ has a unique zero $x_{1} \in(0, \infty)$ such that $g_{8}(x)<0$ for $x \in\left(0, x_{1}\right)$ and $g_{8}(x)>0$ for $x>x_{1}$, so that the piecewise monotoneity of $g_{2}$ follows from (3.7).

By [AS, 6.3.7 and 6.3.8], we have

$$
\begin{aligned}
\psi\left(\frac{2}{3}\right)-\psi\left(\frac{1}{6}\right) & =\psi\left(1-\frac{1}{3}\right)-\psi\left(\frac{1}{6}\right) \\
& =\pi \cot \frac{\pi}{3}+\psi\left(\frac{1}{3}\right)-\psi\left(\frac{1}{6}\right) \\
& =\pi \cot \frac{\pi}{3}+\left[\frac{1}{2} \psi\left(\frac{1}{6}\right)+\frac{1}{2} \psi\left(\frac{1}{6}+\frac{1}{2}\right)+\log 2\right]-\psi\left(\frac{1}{6}\right) \\
& =\pi \cot \frac{\pi}{3}+\frac{1}{2}\left[\psi\left(\frac{2}{3}\right)-\psi\left(\frac{1}{6}\right)\right]+\log 2,
\end{aligned}
$$

so that

$$
\psi\left(\frac{2}{3}\right)-\psi\left(\frac{1}{6}\right)=2 \pi \cot \frac{\pi}{3}+\log 4
$$


Consequently, by [AS, 6.3.2, 6.3.4 and 6.3.5],

$$
\begin{aligned}
g_{8}\left(\frac{3}{2}\right) & =3\left[\psi\left(\frac{3}{2}\right)-\psi(2)\right]-2 \log \frac{\sqrt{\pi} \Gamma(3 / 2)}{2 \Gamma(2)}+\psi\left(\frac{10}{6}\right)-\psi\left(\frac{7}{6}\right) \\
& =3(1-\log 4)-2 \log \frac{\pi}{4}+\psi\left(\frac{2}{3}+1\right)-\psi\left(\frac{1}{6}+1\right) \\
& =-\frac{3}{2}-\log 4-2 \log \pi+\psi\left(\frac{2}{3}\right)-\psi\left(\frac{1}{6}\right) \\
& =-\frac{3}{2}-2 \log (2 \pi)+2 \pi \cot \frac{\pi}{3}+\log 4=-0.16186 \cdots<0 .
\end{aligned}
$$

On the other hand, by [AS, 6.3.2, 6.3.4, 6.1.12, 6.3.5 and 6.3.8],

$$
\begin{aligned}
g_{8}(2) & =4\left(\log 4-\frac{5}{3}\right)-2 \log \frac{2}{3}+\left[\psi\left(\frac{5}{8}\right)+\frac{8}{5}\right]-\left[8+\psi\left(\frac{1}{8}\right)\right] \\
& =3 \log 4+2 \log 3-\frac{196}{15}+\psi\left(\frac{5}{8}\right)-\psi\left(\frac{1}{8}\right) \\
& =3 \log 4+2 \log 3-\frac{196}{15}+\psi\left(\frac{1}{8}+\frac{1}{2}\right)-\psi\left(\frac{1}{8}\right) \\
& =2 \log 12-\frac{196}{15}+2\left[\psi\left(\frac{1}{4}\right)-\psi\left(\frac{1}{8}\right)\right] .
\end{aligned}
$$

It follows from [GR 8.366.4 and 8.363.6, pp. 944-945] that

$$
\psi\left(\frac{1}{4}\right)=-\gamma-\frac{\pi}{2}-3 \log 2
$$

and

$$
\begin{aligned}
\psi\left(\frac{1}{8}\right) & =-\gamma-3 \log 2-\frac{\pi}{2} \cot \frac{\pi}{8}+\sum_{k=1}^{7}\left(\cos \frac{k \pi}{4}\right) \log \left(2 \sin \frac{k \pi}{8}\right) \\
& =-\gamma-4 \log 2-\frac{\pi}{2} \cot \frac{\pi}{8}+\sqrt{2} \log \frac{\sin (\pi / 8)}{\sin (3 \pi / 8)} \\
& =-\gamma-4 \log 2-\frac{\pi}{2} \cot \frac{\pi}{8}+\sqrt{2} \log \tan \frac{\pi}{8},
\end{aligned}
$$

so that

$$
\psi\left(\frac{1}{4}\right)-\psi\left(\frac{1}{8}\right)=-\frac{\pi}{2}+\log 2+\frac{\pi}{2} \cot \frac{\pi}{8}+\sqrt{2} \log \cot \frac{\pi}{8},
$$

and hence,

$$
g_{8}(2)=3 \log 4+2 \log 3-\frac{196}{15}-\pi+\pi \cot \frac{\pi}{8}+2 \sqrt{2} \log \cot \frac{\pi}{8}=0.225224 \cdots>0 .
$$

Consequently, $x_{1} \in(3 / 2,2)$.

Clearly,

$$
g_{2}\left(\frac{1}{2}\right)=\frac{\sqrt{\pi}}{2} \cdot \frac{\pi}{2} \cdot \frac{\Gamma(1 / 2)}{\Gamma(1)}=\frac{\pi^{2}}{4} .
$$

It follows from [AS 6.1.37] that $\lim _{x \rightarrow \infty} g_{2}(x)=2$. 
(3) Logarithmic differentiation gives

$$
\begin{aligned}
& \frac{4 x^{2} g_{3}^{\prime}(x)}{g_{3}(x)}=g_{9}(x) \equiv g_{8}(x)+\frac{2}{2 x+1}-2-\log 4 \\
& =2 x\left[\psi(x)-\psi\left(x+\frac{1}{2}\right)\right]+2 \log \frac{\Gamma(x+1 / 2)}{\sqrt{\pi} \Gamma(x)}+\psi\left(\frac{1}{4 x}+\frac{1}{2}\right)-\psi\left(\frac{1}{4 x}\right)-4 x .
\end{aligned}
$$

By [AS, 6.4.1], for each $x>0$,

$$
\begin{aligned}
g_{8}^{\prime}(x) & =2 x \int_{0}^{\infty} \frac{t e^{-x t} d t}{1+e^{-t / 2}}+\frac{1}{4 x^{2}} \int_{0}^{\infty} \frac{t e^{-[1+1 /(4 x)] t} d t}{1+e^{-t / 2}} \\
& >2 x \int_{0}^{\infty} \frac{t e^{-x t} d t}{1+e^{-t / 2}}=\frac{2}{x} \int_{0}^{\infty} \frac{(x t) e^{-x t} d(x t)}{1+e^{-t / 2}} \\
& =\frac{2}{x} \int_{0}^{\infty} \frac{u e^{-u} d u}{1+e^{-u /(2 x)}}>\frac{1}{x} \int_{0}^{\infty} u e^{-u} d u=\frac{1}{x}
\end{aligned}
$$

and hence,

$$
g_{9}^{\prime}(x)=g_{8}^{\prime}(x)-\frac{4}{(2 x+1)^{2}}>\frac{1}{x}-\frac{4}{(2 x+1)^{2}}=\frac{4 x^{2}+1}{x(2 x+1)^{2}}>0,
$$

so that $g_{9}$ is strictly increasing on $(0, \infty)$. Since $g_{9}\left(0^{+}\right)=-\infty$ and $\lim _{x \rightarrow \infty} g_{9}(x)=\infty$, $g_{9}$ has a unique zero $x_{2}$ such that $g_{9}(x)<0$ for $x \in\left(0, x_{2}\right)$ and $g_{9}(x)>0$ for $x \in\left(x_{2}, \infty\right)$. This, together with (3.9), yields the piecewise monotoneity of $g_{3}$.

Applying the formulas [AS, 6.3.2, 6.3.4, 6.3.5 and 6.1.12]

$$
\begin{aligned}
\psi(n) & =-\gamma+\sum_{k=1}^{n-1} \frac{1}{k}, \psi\left(n+\frac{1}{2}\right)=-\gamma-\log 4+2 \sum_{k=1}^{n} \frac{1}{2 k-1}, \\
\Gamma\left(n+\frac{1}{2}\right) & =\frac{1 \cdot 3 \cdot 5 \cdots(2 n-1)}{2^{n}} \sqrt{\pi},
\end{aligned}
$$

and [GR, 8.370 and 8.372]

$$
\psi\left(\frac{x+1}{2}\right)-\psi\left(\frac{x}{2}\right)=2 \sum_{k=0}^{\infty} \frac{(-1)^{k}}{x+k},
$$

we get

$$
\begin{aligned}
\frac{1}{2 n} g_{9}(n) & =g_{10}(n) \equiv \log 2-2+\frac{1}{n} \log \frac{(2 n-1) ! !}{(n-1) !} \\
& +2 \sum_{k=0}^{\infty} \frac{(-1)^{k}}{2 n k+1}-\sum_{k=1}^{n} \frac{1}{k(2 k-1)}-\frac{1}{n}
\end{aligned}
$$

for $n \in \mathbb{N}$, and hence, by the properties of the Leibniz series and by computation,

$$
\begin{aligned}
g_{10}(33) & =\log 2-2+\frac{1}{33} \log \frac{65 ! !}{32 !}+2 \sum_{k=0}^{\infty} \frac{(-1)^{k}}{66 k+1}-\sum_{k=1}^{33} \frac{1}{k(2 k-1)}-\frac{1}{33} \\
& <\log 2-2+\frac{1}{33} \log \frac{65 ! !}{32 !}+2 \sum_{k=0}^{30000} \frac{(-1)^{k}}{66 k+1}-\sum_{k=1}^{33} \frac{1}{k(2 k-1)}-\frac{1}{33} \\
& =-0.000381 \cdots<0
\end{aligned}
$$


and

$$
\begin{aligned}
g_{10}(34) & =\log 2-2+\frac{1}{34} \log \frac{67 ! !}{33 !}+2 \sum_{k=0}^{\infty} \frac{(-1)^{k}}{68 k+1}-\sum_{k=1}^{34} \frac{1}{k(2 k-1)}-\frac{1}{34} \\
& >\log 2-2+\frac{1}{34} \log \frac{67 ! !}{33 !}+2 \sum_{k=0}^{30001} \frac{(-1)^{k}}{68 k+1}-\sum_{k=1}^{34} \frac{1}{k(2 k-1)}-\frac{1}{34} \\
& =0.000065 \cdots>0 .
\end{aligned}
$$

Hence $x_{2} \in(33,34)$.

Finally, it is clear that $g_{3}(1 / 2)=4 g_{2}(1 / 2)=\pi^{2}$ and $\lim _{x \rightarrow \infty} g_{3}(x)=\lim _{x \rightarrow \infty} g_{2}(x)=2$.

3.13. Proof of Theorem 1.17. (1) By the expression

$$
I_{n}=\frac{1}{2} B\left(\frac{n-1}{2}, \frac{1}{2}\right)
$$

$F_{1}(n)$ can be rewritten as

$$
F_{1}(n)=2 n-4\left[\frac{\Gamma\left(\frac{n}{2}\right)}{\Gamma\left(\frac{n-1}{2}\right)}\right]^{2}=4 f_{4}\left(\frac{n-1}{2}\right)+2
$$

by (1.1), where $f_{4}$ is as in Theorem 1.12. Hence the monotoneity of $F_{1}$ and the limiting value $\lim _{n \rightarrow \infty} F_{1}(n)=3$ follow from Theorem 1.12(2). Clearly, $F_{1}(1)=6-\pi$. Thus, for $n \geq 3,6-\pi \leq F_{1}(n)<3$, so that (1.18) holds.

(2) By (1.2),

$$
F_{2}(n)=2\left\{\frac{n-1}{2}-\left[\frac{\Gamma\left(\frac{n-1}{2}+\frac{1}{2}\right)}{\Gamma\left(\frac{n-1}{2}\right)}\right]^{2}+\frac{1}{2}\right\}=2 f_{4}\left(\frac{n-1}{2}\right)+1,
$$

which is strictly increasing for $n \geq 3$ with $F_{2}(3)=3-\pi / 2$ and $\lim _{n \rightarrow \infty} F_{2}(n)=3 / 2$ by Theorem 1.12(2). Hence the result follows.

(3) It follows from (1.2) that

$$
\frac{\Omega_{n-1}}{\Omega_{n}}=\frac{1}{\sqrt{\pi}} \frac{\Gamma\left(\frac{n}{2}+1\right)}{\Gamma\left(\frac{n+1}{2}\right)}=\frac{\omega_{n}}{\omega_{n+1}},
$$

so that

$$
F_{3}(n)=n-2 \pi\left(\frac{\omega_{n}}{\omega_{n+1}}\right)^{2}=F_{2}(n+2)-2 .
$$

Hence part (3) follows from part (2).

(4) It follows from (1.2), the expression AVV1, (2.32), p. 41]

$$
J_{n}=\frac{1}{2} B\left(\frac{1}{2(n-1)}, \frac{1}{2}\right)
$$

and [AS, 6.1.15] that

$$
b_{n}=\frac{\sqrt{\pi}}{2(n-1)} \frac{\Gamma\left(\frac{1}{2(n-1)}\right)}{\Gamma\left(\frac{1}{2(n-1)}+\frac{1}{2}\right)}=\sqrt{\pi} F_{6}\left(\frac{1}{2(n-1)}\right),
$$


where $F_{6}(x)=\Gamma(x+1) / \Gamma(x+1 / 2)$. Since

$$
F_{6}^{\prime}(x)=F_{6}(x)[\psi(x+1)-\psi(x+1 / 2)]>0
$$

by the monotoneity of $\psi(x), F_{6}$ is strictly increasing on $(0, \infty)$. Hence the monotoneity of $b_{n}$ follows from (3.15), and

$$
1=\sqrt{\pi} F_{6}(0)<b_{n} \leq b_{2}=\pi / 2 .
$$

Next, let $g_{1}$ be as in Theorem 1.16(1). Then by (1.4) and (3.14),

$$
B_{n}=\left[\frac{1}{2(n-1)} B\left(\frac{1}{2(n-1)}, \frac{1}{2}\right)\right]^{n-1}=\sqrt{g_{1}\left(\frac{1}{2(n-1)}\right)},
$$

and hence the monotoneity of $B_{n}$ follows from Theorem 1.16(1). Moreover, for $n \geq 2$,

$$
\frac{\pi}{2}=B_{2} \leq B_{n}<\lim _{n \rightarrow \infty} B_{n}=\sqrt{g_{1}\left(0^{+}\right)}=2 .
$$

The second double inequality in (1.21) follows from (3.16) and (3.17).

(5) We know that $n-J_{n}-a$ has the expression

$$
n-J_{n}-a=\int_{0}^{\pi / 2}\left[1-(\sin t)^{1 /(n-1)}\right] \tan \frac{t}{2} d t .
$$

(Cf. [AVV1, p. 43].) Hence

$$
F_{4}(n)=\int_{0}^{\pi / 2} F_{7}\left(\frac{1}{n-1}, t\right) \tan \frac{t}{2} d t,
$$

where $F_{7}(x, t)=\left[1-(\sin t)^{x}\right] / x$. Since the function

$$
F_{8}(x, t) \equiv \frac{\frac{d}{d x}\left[1-(\sin t)^{x}\right]}{\frac{d}{d x}(x)}=(\sin t)^{x} \log \frac{1}{\sin t}
$$

is strictly decreasing in $x$ on $(0, \infty)$ for each fixed $t \in(0, \pi / 2)$, it follows from the Monotone l'Hôpital's Rule [AVV1 Theorem 1.25] that $F_{7}$ is strictly decreasing in $x$ on $(0, \infty)$. Hence $F_{4}$ is strictly increasing for $n \geq 3$.

Clearly, $F_{4}(3)=2\left(3-J_{3}-a\right)=2(b-a)$. Set $x=1 /[2(n-1)]$. Then, by $(3.14)$,

$$
\begin{aligned}
F_{4}(n) & =\frac{1}{2 x}\left[\frac{1}{2 x}+1-\frac{1}{2} B\left(x, \frac{1}{2}\right)-a\right] \\
& =\frac{1}{4 x^{2}}\left[x \log 4-\sqrt{\pi} \frac{\Gamma(x+1)}{\Gamma\left(x+\frac{1}{2}\right)}+1\right] .
\end{aligned}
$$


Hence, by l'Hôpital's Rule,

$$
\begin{aligned}
F_{4}(\infty) \equiv & \lim _{n \rightarrow \infty} F_{4}(n) \\
= & \frac{1}{4} \lim _{x \rightarrow 0} \frac{x \log 4+1-[\sqrt{\pi} \Gamma(x+1) / \Gamma(x+1 / 2)]}{x^{2}} \\
= & \frac{1}{8} \lim _{x \rightarrow 0} \frac{1}{x}\left\{\log 4-\sqrt{\pi} \frac{\Gamma(x+1)}{\Gamma\left(x+\frac{1}{2}\right)}\left[\psi(x+1)-\psi\left(x+\frac{1}{2}\right)\right]\right\} \\
= & -\frac{\sqrt{\pi}}{8} \lim _{x \rightarrow 0} \frac{\Gamma(x+1)}{\Gamma\left(x+\frac{1}{2}\right)} \\
& \times\left\{\left[\psi(x+1)-\psi\left(x+\frac{1}{2}\right)\right]^{2}+\left[\psi^{\prime}(x+1)-\psi^{\prime}\left(x+\frac{1}{2}\right)\right]\right\} \\
= & -\frac{1}{8}\left[(\log 4)^{2}+\frac{\pi^{2}}{6}-\psi^{\prime}\left(\frac{1}{2}\right)\right]=\frac{1}{8}\left[\frac{\pi^{2}}{3}-(\log 4)^{2}\right]=c .
\end{aligned}
$$

The inequality (1.22) is clear.

(6) By (1.2), (1.4), (1.5) and [AS, 6.1.15], we have

$$
A_{n}=\left[\frac{\pi^{n / 2} \Gamma\left(\frac{n-1}{2}\right)}{2^{n} \Gamma\left(\frac{n}{2}\right)}\right]^{1 /(n-1)} \frac{\Gamma\left(\frac{1}{2(n-1)}\right)}{\Gamma\left(\frac{1}{2(n-1)}+\frac{1}{2}\right)} .
$$

Putting $x=(n-1) / 2$ and applying [AS, 6.1.15], we get

$$
F_{5}(n)=\frac{\sqrt{\pi}}{2} \frac{1}{2 x+1}\left[\frac{\sqrt{\pi}}{2} \frac{\Gamma(x)}{\Gamma\left(x+\frac{1}{2}\right)}\right]^{1 /(2 x)} \frac{\Gamma\left(\frac{1}{4 x}\right)}{\Gamma\left(\frac{1}{4 x}+\frac{1}{2}\right)}=\frac{1}{2} g_{2}(x),
$$

where $g_{2}$ is as in Theorem 1.16(2).

Next, computation gives

$$
F_{5}(4)=\frac{\sqrt{\pi}}{2}\left[\frac{\sqrt{\pi}}{2} \frac{\Gamma(3 / 2)}{\Gamma(2)}\right]^{1 / 3} \frac{\Gamma(1+1 / 16)}{\Gamma(1+9 / 16)}=0.8890941475 \ldots
$$

and

$$
F_{5}(5)=\frac{\sqrt{\pi}}{2}\left[\frac{\sqrt{\pi}}{2} \frac{\Gamma(2)}{\Gamma(2+1 / 2)}\right]^{1 / 4} \frac{\Gamma(1+1 / 8)}{\Gamma(1+5 / 8)}=0.8411396629 \cdots .
$$

The result for $F_{5}$ now follows from (3.19), (3.20), (3.21) and Theorem 1.16(2).

The limiting value $\lim _{n \rightarrow \infty}(1 / n) A_{n}=1$ follows from Theorem 1.16(2), and the inequality in (1.23) is clear.

(7) Put $x=(n-1) / 2$. Then, by (3.19),

$$
D_{n}=2^{n /(n-1)} \frac{1}{n-1} A_{n}=2^{n /(n-1)} \frac{n}{n-1} \cdot \frac{A_{n}}{n}=g_{3}(x),
$$


where $g_{3}$ is as in Theorem 1.16(3). Hence, by Theorem 1.16(3), $D_{n}$ is strictly decreasing for $2 \leq n \leq 67$ and strictly increasing for $n \geq 69$.

By computation, we obtain

$$
\begin{aligned}
D_{67} & =\frac{2^{67 / 66}}{66} A_{67}=\frac{1}{66}\left\{\pi^{67 / 2} \frac{\Gamma(33)}{\Gamma(33+1 / 2)}\right\}^{1 / 66} \frac{\Gamma(1 / 132)}{\Gamma(67 / 132)} \\
& =\frac{\sqrt{2 \pi}}{66}\left(\frac{32 !}{65 ! !}\right)^{1 / 66} \frac{\Gamma(1 / 132)}{\Gamma(67 / 132)}=1.98535339227 \cdots \\
D_{68} & =\frac{2^{68 / 67}}{67} A_{68}=\frac{1}{67}\left\{\pi^{68 / 2} \frac{\Gamma(33+1 / 2)}{\Gamma(34)}\right\}^{1 / 67} \frac{\Gamma(1 / 134)}{\Gamma(68 / 134)} \\
& =\frac{1}{67} \frac{\pi^{69 / 134}}{2^{33 / 67}}\left(\frac{65 ! !}{33 !}\right)^{1 / 67} \frac{\Gamma(1 / 134)}{\Gamma(68 / 134)}=1.98534941481 \cdots \\
D_{69} & =\frac{2^{69 / 68}}{68} A_{69}=\frac{1}{68}\left\{\pi^{69 / 2} \frac{\Gamma(34)}{\Gamma(34+1 / 2)}\right\}^{1 / 68} \frac{\Gamma(1 / 136)}{\Gamma(69 / 136)} \\
& =\frac{\sqrt{2 \pi}}{68}\left(\frac{33 !}{67 ! !}\right)^{1 / 68} \frac{\Gamma(1 / 136)}{\Gamma(69 / 136)}=1.98534877792 \cdots, \\
D_{70} & =\frac{2^{70 / 69}}{69} A_{70}=\frac{1}{69}\left\{\pi^{70 / 2} \frac{\Gamma(34+1 / 2)}{\Gamma(35)}\right\}^{1 / 69} \frac{\Gamma(1 / 138)}{\Gamma(70 / 138)} \\
& =\frac{1}{69} \frac{\pi^{71 / 138}}{2^{34 / 69}}\left(\frac{67 ! !}{34 !}\right)^{1 / 69} \frac{\Gamma(1 / 138)}{\Gamma(70 / 138)}=1.98535124396 \cdots
\end{aligned}
$$

These values, together with the conclusion just proved above, yield the piecewise monotoneity of $D_{n}$.

Clearly, $D_{2}=\pi^{2}$. By (3.22) and (1.23), $\lim _{n \rightarrow \infty} D_{n}=2$. Hence (1.24) holds.

\section{ACKNOWLEDGMENTS}

The authors are indebted to the referee, whose remarks led to several improvements in the final text.

\section{REFERENCES}

[AS] M. Abramowitz and I. A. Stegun, Handbook of Mathematical Functions with Formulas, Graphs and Mathematical Tables, Dover, New York, 1965. MR 34:8606

[A1] H. AlzER, Some gamma function inequalities, Math. Comp., 60(1993), 337-346. MR 93f:33001

[A2] H. Alzer, On some inequalities for the gamma and psi functions, Math. Comp., 66(1997), 373-389. MR 97e:33004

[A3] H. Alzer, Inequalities for the gamma and polygamma functions, Abh. Math. Sem. Univ. Hamburg, 68(1998), 363-372. MR 99k:33002

[A4] H. Alzer: Inequalities for the volume of the unit ball in $\mathbb{R}^{n}$, J. Math. Anal. Appl. 252 (2000), 353-363. MR 2001m:26036

[A5] H. Alzer: On Ramanujan's double inequality for the gamma function (English. English summary), Bull. London Math. Soc. 35 (2003), no. 5, 601-607.

[ABrVV] G. D. Anderson, R. W. Barnard, K. C. Richards, M. K. Vamanamurthy and M. VuORInen, Inequalities for zero-balanced hypergeometric functions, Trans. Amer. Math. Soc., 347(1995), 1713-1723. MR 95m:33002 
[AQ] G. D. Anderson And S.-L. QIU, A monotoneity property of the gamma function, Proc. Amer. Math. Soc., (125)1995, 3355-3362. MR 98h:33001

[AVV1] G. D. Anderson, M. K. Vamanamurthy and M. Vuorinen, Conformal Invariants, Inequalities, and Quasiconformal Maps, John Wiley \& Sons, 1997. MR 98h:30033

[AVV2] G. D. Anderson, M. K. Vamanamurthy, and M. Vuorinen, Topics in special functions, Papers on Analysis: A volume dedicated to Olli Martio on the occasion of his 60th birthday, ed. by J. Heinonen, T. Kilpeläinen, and P. Koskela, Report 83, Univ. Jyväskylä (2001), 5-26, ISBN 951-39-1120-9. (http://www.math.jyu.fi/research/report83.html) MR 2002j:00014

[AAR] G. Andrews, R. Askey, R. Roy, Special Functions, Encyclopedia of Mathematics and its Applications, Vol. 71, Cambridge Univ. Press, 1999. MR 2000g:33001

[BH] J. Böhm And E. Hertel, Polyedergeometrie n-dimensionalen Räumen Konstanter Krümmung, Birkhäuser, Basel-Boston-Stuttgart, 1981. MR 82k:52001a

[BP] C. Berg and H. Pedersen, A completely monotone function related to the gamma function, J. Comp. Appl. Math. 133 (2001), 219-230. MR 2003k:33001

[B] K. H. Borgwardt, The Simplex Method, a Probabilistic Analysis, Springer-Verlag, Berlin, 1987. MR 88k:90110

[EL] Á. Elbert and A. Laforgia, On some properties of the gamma function, Proc. Amer. Math. Soc. 128 (2000), 2667-2673. MR 2000m:33002

[G] W. Gautschi, The incomplete gamma functions since Tricomi. Tricomi's ideas and contemporary applied mathematics (Rome/Turin, 1997), 203-237, Atti Convegni Lincei, 147, Accad. Naz. Lincei, Rome, 1998. MR 2001g:33003

[GR] I. S. Gradshteyn And I. M. Ryzhik, Tables of Integrals, Series, and Products, 4th ed., prepared by Yu. V. Geronimus and M. Yu. Tseytlin, Academic Press, New YorkLondon, 1965. MR 33:5952

[K1] E. A. Karatsuba, On the computation of the Euler constant $\gamma$, Numer. Algorithms 24 (2000), 83-87. MR 2002f:33004

[K2] E. A. Karatsuba: On the asymptotic representation of the Euler gamma function by Ramanujan, J. Comp. Appl. Math. 135.2 (2001), 225-240. MR 2002i:33004

[Ke] D. Kershaw, Some extensions of W. Gautschi's inequalities for the gamma function. Math. Comp. 41 (1983), no. 164, 607-611. MR 84m:33003

[KR] D. A. Klain And G.-C. Rota, A continuous analogue of Sperner's Theorem, in Communications on Pure and Applied Mathematics, Vol. L, John Wiley \& Sons, 1997, pp. 205-223. MR 98c:05155

[L] A. Laforgia, Further inequalities for the gamma function, Math. Comp. 42 (1984), no. 166, 597-600. MR 85i:33001

[LL] I. B. LAZAREviĆ AND A. LupAS, Functional equations for Wallis and gamma functions, Univ. Beograd. Publ. Elektrotehn. Fak. Ser. Electr. Telec. Autom, 461-497(1974), 245251. MR 50:13631

[Me1] M. MERKLE, Logarithmic convexity and inequalities for the gamma function, J. Math. Anal. Appl., 203(1996), 369-380. MR 98c:33001

[Me2] M. MerkLe, Convexity, Schur-convexity and bounds for the gamma function involving the digamma function, Rocky Mountain J. Math. 28 (1998), no. 3, 1053-1066. MR 99m:33001

[Me3] M. MERKLE, Conditions for convexity of a derivative and some applications to the gamma function, Aequationes Math. 55 (1998), no. 3, 273-280. MR 99d:33002

[Mi] D. S. Mitrinović, Analytic Inequalities, Grundlehren Math. Wiss., Band 165, Springer-Verlag, Berlin, 1970. MR 43:448

[MSC] D. S. Mitrinović, J. Sańdor and B. Crstici, Handbook of Number Theory, Kluwer Acad. Publ., 1995. MR 97f:11001

[S] J. SAndor, Sur la fonction gamma, Publ. Centre Rech. Math. Pures (I) (Neuchâtel), 21(1989), 4-7.

[T] N. M. Temme, Special functions. An introduction to the classical functions of mathematical physics, A Wiley-Interscience Publication, John Wiley \& Sons, Inc., New York, 1996. MR 97e:33002

[TT] S. R. Tims And J. A. Tyrrell, Approximate evaluation of Euler's constant, Math. Gaz., 55(1971), 65-67. MR 58:10710 
[V] J. VÄISÄLÄ, Lectures on n-Dimensional Quasiconformal Mappings, Lecture Notes in Math., Vol.229, Springer-Verlag, Berlin, 1971. MR 56:12260

[Vu] M. Vuoninen, Conformal Geometry and Quasiregular Mappings, Lecture Notes in Math., Vol. 1319, Springer-Verlag, Berlin, 1988. MR 89k:30021

[WW] E. T. Whittaker and G. N. Watson, A Course of Modern Analysis, 4th ed., Cambridge Univ. Press, London, 1958. MR 31:2375

[Y] R. M. YounG, Euler's constant, Math. Gaz., 75(1991), 187-190.

President's Office, Hangzhou Institute of Electronics Engineering (HieE), HangZhou 310037, Peoples Republic of China

E-mail address: sl_qiu@hziee.edu.cn

Department of Mathematics, University of Turku, Vesilinnankatu 5, Fin-20014, Turku, Finland

E-mail address: vuorinen@csc.fi 